
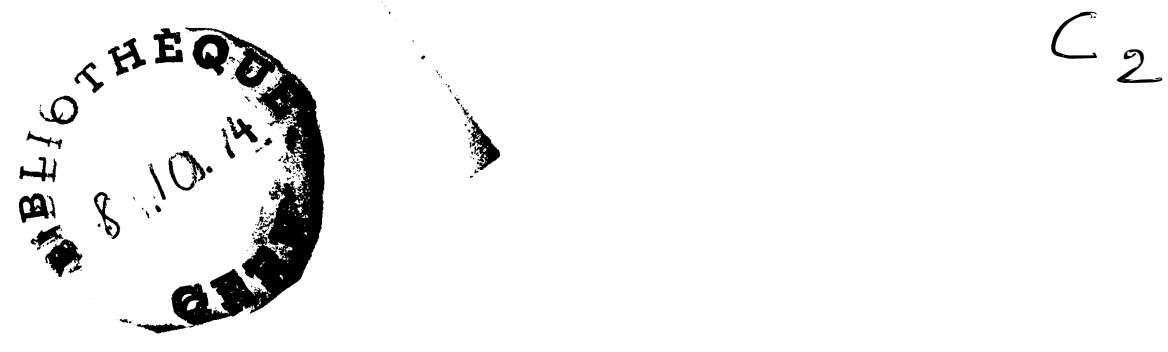

EUROPEAN ORGANISATION FOR NUCLEAR RESEARCH

CERN-ISR-VA/74-44

Closed distribution

\title{
A MINIATURE EXTRACTOR GAUGE FOR THE UHV
}

by

D. Blechschmidt

\begin{abstract}
The design and performance of a miniaturized hot filament ionization pressure gauge, specially developed for the CERN Intersecting Storage Rings, is described. It is mounted on a standard metal flange with ceramic feedthroughs and fits into an aperture of $15.7 \mathrm{~mm}$ (less than $2 / 3$ inch). The gauge is of the extractor type and has a sensitivity for nitrogen of about $5 \mathrm{Torr}^{-1}$. Its low pressure limit, about $5 \times 10^{-12}$ Torr nitrogen equivalent, is mainly due to $x-r$ ays. These two characteristic figures have been obtained, in spite of the gauge's smallness, by an optimization of most of the geometrical and electronical parameters. Some problems, seriously affecting the gauge's performance, and particularly arising from its size, such as space charge effects and thermal outgassing, are discussed.

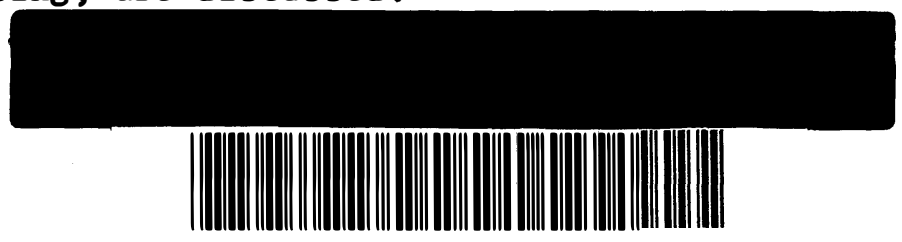

CM-P00064824

Geneva, 27 th August 1974
\end{abstract}

* Paper to be submitted for publication to the Journal of Vacuum Science and Technology. 


\section{INTRODUCTION}

The CERN Intersecting Storage Rings represent the largest ultrahigh vacuum system to date ${ }^{1)}$ with an average pressure of less than $10^{-11}$ Torr. The size of vacuum components, however, incorporated in its eight crossing regions, has to be as small and light as possible in order to minimize the scattering of secondary particles poduced by the colliding proton beams. The vacuum in a chamber enclosing the colliding beams has to be extremely good : it is typically in the low $10^{-12}$ Torr range, so as to reduce the number of spurious events which are caused by nuclear interactions of protons with residual gas molecules.

Consequently, a gauge for pressure measurements in such a crossing region has to be as small as it can possibly be made. Its sensitivity, however, has to be high enough to provide a measurable collector current together with a low pressure limitation which is at least in the $10^{-12}$ Torr range. To the author's knowledge, a gauge with these facilities has not yet been accomplished. Obviously, gauges using a magnetic field are not suitable for miniaturization due to a magnet's bulkiness. The orbitron ionization gauge is also excluded since it is principally limited by space charge effects at about $2 \times 10^{-11}$ Torr already ${ }^{2}$.

The nitrogen sensitivity of an ionization gauge operating at an electron energy of about $100 \mathrm{eV}$ may be estimated from ${ }^{3}$ )

$$
S \approx 10 \cdot \lambda \operatorname{Torr}^{-1}
$$

where $\lambda$ denotes the average distance in $\mathrm{cm}$ that an electron travels within the ionization volume. Since $\lambda$ is approximately the diameter of this volume, the reduction of the gauge dimensions results necessarily in a lower sensitivity. The gauge's low pressure limit due to $x$-rays

$$
P_{X}=Y_{a} Y_{c} G / S
$$

thus increases with its smallness. In this equation; $\mathrm{Y}_{\mathrm{a}}$ denotes the number of $x$-rays emitted from the anode per incident electron, $Y_{c}$ is the 
number of electrons released from the collector per absorbed $\mathrm{x}-\mathrm{r}$ ay and $\mathrm{G}$ is the fraction of $x-r a y$ flux from the anode that is intercepted by the collector $^{4)}$.

Of course, the size of a total pressure gauge has in principle no effect on the geometrical factor, as $G$ is usually called. The collector, however, cannot be made of any small diameter due to mechanical reasons and in order to avoid a pressure dependent sensitivity as observed in some Bayard-Alpert gauges with very fine ion collectors 5 ) Thus, with a lower sensitivity and a higher geometrical factor, the x-ray limit of a miniaturized gauge is inevitably higher than that of a normal one, see Equ. (2).

Amongst the different types of ionization gauges that might be adopted for miniaturization, the extractor gauge as developed by Redhead ${ }^{6)}$ and later improved by Barz and Kocian ${ }^{7)}$, probably has the smallest G/S ratio and thus the lowest $x-r a y$ limit. Furthermore, the residual current caused by electronic desorption of ions from chemisorbed gases on the grid - another important process limiting an ionization gauge at low pressures - is excellently suppressed in the extractor gauge 6,8). Finally, this type of gauge is of relatively simple design, making it easier to solve the technical problems arising from miniaturization.

Figure 1 is a photograph of the miniature extractor gauge (MEG) which is described in this paper. So far, it is the smallest gauge with a low pressure limitation in the $10^{-12}$ Torr range. Its design tolerances are extremely small to provide mechanical solidity together with stable electronic characteristics. The gauge head, for example, has to sustain a temperature of some $800^{\circ} \mathrm{C}$ during degassing by electron bombardment; the entire gauge must withstand a bakeout at $400^{\circ} \mathrm{C}$ without being distorted mechanically. The power input is minimized during normal operation to prevent intrinsic outgassing which causes false pressure readings. Another big problem, arising from the MEG's size, are space charges which influence the gauge's performance drastically. 


\section{CONSTRUCTION}

Figure 2 is a cutaway drawing of the MEG. Electrons are emitted from a thoria coated tungsten filament and accelerated towards the anode grid cage which is made by photoetching from a $0.16 \mathrm{~mm}$ tungsten sheet. Positive ions, produced from residual gas molecules within the anode grid, which is closed at its top, are extracted through the aperture in an electrode closing its base. The purpose of this electrode is to protect the ion collector from $x$-rays which are emitted from the anode and to extract the ions. Therefore, it is referred to as the shield or extraction electrode. The ions are then collected by a very thin and short tungsten wire onto which they are focussed by a semi-spherical reflector electrode.

All relevant dimensions of the MEG are compiled in Table I, the notations used therein are explained in Figure 3. The gauge head is supported by three pairs of $40 \mathrm{~mm}$ long bars; this length was chosen to provide the distance of the gauge head from the wall of the vacuum chamber as required for the ISR. These supports are mounted diametrically around the central rod which keeps the ion collector in position and they supply the filament, the shield and reflector electrode. The anode grid is fixed to the reflector electrode for practical reasons: both electrodes may be operated at the same potential, since experiments with several MEG prototypes have proved that the performance of the gauge is not affected in this mode. The bars are made of stainless steel, except for the filament supports which are tungsten rods of $1 \mathrm{~mm}$ diameter. The collector support is protected against $x$-rays by a thin-walled stainless steel tube at ground potential. Two alumina discs, one mounted just below the gauge's head and the other just above the flange, keep the electrodes well aligned in the gauge head. The cathode is a circular tungsten filament surrounding the anode grid, (see Fig. 1). Since its two $180^{\circ}$ sectors are heated parallel, they provide a better security against burning out. All electrodes, except for the ion collector, may be degassed, not only by electron bombardment at $45 \mathrm{~mA}$ and $700 \mathrm{~V}$ but also by direct ohmic heating in this mounting. 
Prior to assembly, all parts of the gauge are baked at $1000-1050$ ${ }^{\circ} \mathrm{C}$ and at a pressure of below $10^{-5}$ Torr for at least two hours, except for the flange with its feedthroughs which is treated at $450^{\circ} \mathrm{C}$ for about ten hours. This procedure proved to be necessary to decrease the gauge's intrinsic outgassing rate. The seven ceramic feedthroughs are welded onto the $21 \mathrm{~mm}$ o.d. metal flange by electron beam welding techniques. The maximum diameter of the gauge head is $15.3 \mathrm{~mm}$.

\section{LIMITATIONS}

The greatest difficulty encountered during the development of the MEG was the achievement of an ultimate low pressure limit, a maximum of sensitivity and a high electron emission current at the same time. Inevitably, the result is a compromise between these requirements.

A. X-ray limit

An estimation of the $x$-ray limit is already given in Equ. (2). The geometrical factor used therein may be calculated from Redhead's expression for an extractor gauge ${ }^{6)}$

$G=\frac{2 A_{c}}{\pi A_{g}}\left[(1-a) /\left(1+a^{2}\right)^{\frac{1}{2}}-b /\left(a^{2}+b^{2}\right)^{\frac{1}{2}}+\ln \left\{a+\left(1+a^{2}\right)^{\frac{1}{2}}\right\}\right]$

where $A_{g}$ and $A_{c}$ are the surface areas of grid and collector respectively. The parameters $a=r_{g} / h$ and $b=x / h$ are explained in Figure 3. Using the data as listed in Table $I, G=1.5 \times 10^{-4}$ is found from Equ. (3) and $S=9 \operatorname{Torr}^{-1}$ with $\lambda=2 \mathrm{r}_{\mathrm{g}}$ from Equ. (1). Hence, one obtains an $\mathrm{x}$-rạy limit of $3 \times 10^{-12}$ Torr from Equ. (2), using $\mathrm{Y}_{\mathrm{a}}=10^{-5} \mathrm{x}$-rays/ electron ${ }^{9)}$ and a photoelectric yield $Y_{c}=2 \times 10^{-2}$ electron/x-ray $\left.{ }^{10}\right)$ for an anode-to-cathode potential of about $100 \mathrm{~V}$.

However, not all the electrons have their optimal pathlength $\lambda=2 r_{g}$ within the grid cage, since many of them are defocussed by electric field distortions in the cathode's vicinity, caused by space charges or an incorrect filament bias. Furthermore, the ions are not all extracted from the ionizing volume and only part of them are collected. 
Hence, the sensitivity is in fact considerably smaller and the figure obtained as the $\mathrm{x}$-ray limit is rather too low.

\section{B. Electronic desorption}

The second important limitation at low pressures is due to electron induced ion desorption from the anode. Whereas the $x$-ray limit is mainly determined by the gauge's geometry, sensitivity and the electron energy, the electronic desorption is governed by the amount and the nature of chemisorbed gases, the electron current density at the anode surface and by the grid temperature. The low pressure performance of the gauge is far less influenced by electronic ion desorption than in Bayard-Alpert or suppressor gauges ${ }^{6,8}$. Since the MEG is normally operated at 5 to $10 \mathrm{~mA}$, i.e. at the same emission current as in a gauge of standard size, an even higher electron current density at the anode is provided, resulting in a cleaner grid. The MEG's ion desorption limit is thus expected to be lower than of a normal size extractor gauge, where it is in the low $10^{-13}$ Torr range $e^{4,6)}$ and therefore negligible compared with the $x-r a y$ limit.

\section{Intrinsic outgassing}

The first MEG prototypes were warming up considerably during normal operation, giving rise to thermal induced outgassing from the gauge and from the walls of the surrounding vacuum system. Consequently, the power input had to be reduced. By painting a thin layer of $\mathrm{ThO}_{2}$, suspended in n-butanol, onto the filament and flashing it shortly at some $2000^{\circ} \mathrm{C}$ and at a pressure of less than $10^{-6}$ Torr, the gauge does not warm up by more than about $5-10^{\circ} \mathrm{C}$ above ambient temperature. This is also necessary in order to decrease the filament temperature and therewith the tungsten vapour pressure which causes another low pressure limitation $^{11)}$. 


\section{Space charges}

The MEG has a relatively low sensitivity due to its smallness. In order to provide a maximum ion current $I_{c}$ at a given pressure $p$, it has to be operated with a high electron emission $I_{e}$, according to

$$
I_{c}=p S I_{e}
$$

At pressures of less than $10^{-11}$ Torr, for example, an emission of $10 \mathrm{~mA}$ provides an ion current of much less than $10^{-12} \mathrm{~A}$, which is already difficult to measure correctly. However, due to its small dimensions, space charges build up in the MEG between anode and filament and tend to limit the emission current. The maximum space charge limited emission is given by the classical Schottky-Langmuir equation :

$$
I_{e, \max }=2.33 \cdot 10^{-3} \cdot \mathrm{C} \cdot \mathrm{U}_{\mathrm{gf}}{ }^{3 / 2} \cdot \mathrm{A}_{\mathrm{f}} \cdot\left(\mathrm{r}_{\mathrm{f}}-\mathrm{r}_{\mathrm{g}}\right)^{-2} \mathrm{~mA}
$$

where $\mathrm{C} \approx \mathrm{r}_{\mathrm{g}} / \mathrm{r}_{\mathrm{f}}$ is a correction factor that takes into account the circular geometry ${ }^{12}$ ). The filament surface area $A_{f}$ as well as the filament and grid $r$ adii $r_{f}$ and $r_{g}$ are fixed parameters, determined by the gauge's size.

Hence, to provide a maximum emission current, the anode-to-cathode potential $\mathrm{U}_{\mathrm{gf}}$ has to be as high as possible. The ionization cross-section for most residual gases, however, decreases with rising electron energy for $\mathrm{U}_{\mathrm{gf}}$ above $120 \mathrm{~V}$, but the product $\mathrm{Y}_{\mathrm{a}} \mathrm{Y}_{\mathrm{c}}$ increases : i.e. the $\mathrm{x}-\mathrm{ray}$ limit is higher for greater $\mathrm{U}_{\mathrm{gf}}$ values, see Equ. (2). Consequently, the optimum anode-to-cathode voltage for the MEG must be a compromise between a low pressure limitation and a high collector current. A series of MEG prototypes with different $r_{g}$ and $r_{f}$ parameters have been tested. The results were in agreement with Equ. (5), but the maximum emission current which can be achieved is about $11 \mathrm{~mA}$ only for $\mathrm{U}_{\mathrm{gf}}=125 \mathrm{~V}$ and $\mathrm{r}_{\mathrm{g}}=4.5 \mathrm{~mm}$ and $\mathrm{r}_{\mathrm{f}}=6.5 \mathrm{~mm}$. 


\section{RESULTS AND PERFORMANCE}

Many prototypes were tested until finally the MEG model, shown in Figures 1 and 2, proved to be the best. Its geometrical and electronical parameters are compiled in Tables I and II; all measurements hereafter are concerned with these parameters if they are not explicitly mentioned.

The measurements were carried out in an all-metal system with a basic pressure of approximately $2 \times 10^{-12}$ Torr. During the cool-down period, after a bakeout at $350^{\circ} \mathrm{C}$ during 24 hours, the MEG was degassed at $45 \mathrm{~mA}$ with the anode, shield and reflector electrode at $+700 \mathrm{~V}$. A calibrated Helmer gauge was used as a reference; its low pressure limit ( $\mathrm{p}_{1 \mathrm{im}}=2.5 \times 10^{-12}$ Torr) was taken into account.

\section{A. Sensitivity and low pressure limit}

The MEG collector current is plotted against the true pressure for argon, nitrogen and hydrogen in Figure 4. The gas was injected after the system had been pumped down to its basic pressure. The MEG was operated at $10 \mathrm{~mA}$ emission current. From the slope of these three curves, sensitivities of $1.8,5.0$ and 6.3 Torr $^{-1}$, for $\mathrm{H}_{2}, \mathrm{~N}_{2}$ and Ar respectively, have been found, being in rather good agreement with the relative corresponding ionization cross-sections ${ }^{13)}$.

The deviation from the straight line at the low pressure end of the nitrogen injection curve is due to the MEG's residual current of $2.7 \times 10^{-13} \mathrm{~A}$. This figure is obtained as the pressure independent current that has to be subtracted from the measured values to yield a straight line of slope one throughout, i.e. a curve that corresponds to Equ. (4). The MEG's low pressure limit is hence $5.4 \times 10^{-12}$ Torr and as it was already expected, it is slightly higher than the theoretical value.

\section{B. Electron emission current}

The space charge in the vicinity of the cathode tends to force the electrons onto trajectories of lower radial momentum and higher axial drift. Consequently, their pathlength within the grid structure is 
reduced together with the sensitivity of the gauge. A reduction of the emission current results in a slightly higher sensitivity of about 6 Torr ${ }^{-1}$ for $\mathrm{N}_{2}$ and $I_{e}=5 \mathrm{~mA}$, a figure that comes closer to the theoretical value of 9 Torr $^{-1}$. This is shown in Figure 5 where the ion current $I_{c}$ is plotted as a function of the electron emission, at $\mathrm{p}=2 \times 10^{-10^{c}}$ and $1.5 \times 10^{-9}$ Torr injected nitrogen. $I_{c}$ is proportional to the emission as long as $I_{e} \leqslant 5 \mathrm{~mA}$; the sensitivity decreases, however, for higher emission currents. The emission is limited at about $11 \mathrm{~mA}$ as predicted from Equ. (5) for $\mathrm{U}_{\mathrm{gf}}=125 \mathrm{~V}$. Unfortunately, as explained above, $\mathrm{I}_{\mathrm{e}}$ can only be increased for higher $U_{g f}$ values, i.e. if a higher $x-r a y$ limit may be tolerated.

\section{Filament potential}

Space charges are not the only reason why electrons are placed onto unfavourable trajectories : electric field distortions due to an incorrect filament bias have the same effect ${ }^{14}$ ). The cathode potential has to be equal to the cylindrical equipotential surface existing between the anode and the grounded tube wall in which the gauge is mounted. It is given by

$$
U_{f, o p t}=\alpha U_{g f} /(1-\alpha) \text { with } \alpha:=\log \left(r_{t} / r_{f}\right) / \log \left(r_{t} / r_{g}\right)
$$

where $r_{t}$ denotes the inner radius of the tube wall. This condition is much more critical for small tubes than for big ones. An optimal filament potential of about $90 \mathrm{~V}$ results from this equation for $U_{g f}=125 \mathrm{~V}$ and $2 \mathrm{r}_{t}=17 \mathrm{~mm}$, which is the tube diameter in which the MEG still might be required to be operating .

Figure 6 is a plot of the collector current as a function of the anode-to-cathode potential for different filament and shield electrode voltages, $U_{f}$ and $U_{s}$. The MEG was mounted in a tube of $r_{t}=20.5 \mathrm{~mm}$ for this measurement, which was performed at a pressure of $1.7 \times 10^{-9}$ Torr injected nitrogen. It is evident from Equ. (6) that the bigger the tube radius, the higher the filament bias has to be in order to yield the 
optimal performance. A maximum sensitivity would have been found at $\mathrm{U}_{\mathrm{f}}=390 \mathrm{~V}$ for $\mathrm{r}_{\mathrm{t}}=20.5 \mathrm{~mm}$. Nevertheless, the sensitivity at $\mathrm{U}_{\mathrm{f}} \leqslant 150 \mathrm{~V}$ is still fairly high. If, however, the filament is cperated at a bias which is too positive, it represents a potential dip which many electrons cannot leave and a cloud of electron envelops the cathode. This impairs the gauge performance because the electron's radial momentum is reduced and therewith the sensitivity. A filament bias of $100 \mathrm{~V}$ has been chosen from these results; it permits, according to Equ. (6), the operation of the MEG even in a tube of $17.6 \mathrm{~mm}$ diameter.

\section{Shield potential}

The choice of the shield potential $U_{s}$ is less important for the MEG's performance. However, if it is too low, the extraction efficiency of positive ions from within the anode grid volume may be poor, since too many ions are collected by the shield electrode itself. Electrons may, however, travel through the extraction aperture and impinge on the reflector electrode if $U_{S}$ is too high. The true collector current will then be masked by $x$-ray emission and ion desorption from the reflector.

This is shown in Figure 7, where the collector current is plotted against the electron acceleration voltage. The measurements were made at about the MEG's low pressure limit. At this low pressure, the slope of the ion current is much steeper for increasing electron energy than in Figure 6. This is due to the higher contribution of $x$-ray current, rising with a power of about 1.5 with the electron energy ${ }^{9}$ ). Beyond a critical value for $\mathrm{U}_{\mathrm{gf}}$, which is, for example, about $220 \mathrm{~V}$ for $\mathrm{U}_{\mathrm{f}}=25 \mathrm{~V}$ and $\mathrm{U}_{\mathrm{S}}=-50 \mathrm{~V}$, the collector current increases drastically. This is due to ion desorption and $x$-ray emission from the reflector, stimulated by electrons travelling through the extraction aperture.

The potential barrier, which these electrons have to overcome, to get to the reflector, is in a very rough approximation proportional to $\mathrm{U}_{\mathrm{g}}-\mathrm{U}_{\mathrm{S}}$. They do not impinge at the reflector except if :

$$
\mathrm{U}_{\mathrm{f}}-\mathrm{U}_{\mathrm{s}} \ll \beta\left(\mathrm{U}_{\mathrm{g}}-\mathrm{U}_{\mathrm{s}}\right) \text { i.e. } \mathrm{U}_{\mathrm{s}} \ll \mathrm{U}_{\mathrm{f}}-\beta \mathrm{U}_{\mathrm{gf}} /(1-\beta)
$$


From curves as in Figure 7, obtained with different parameters $U_{f}$ and $U_{s}$, $\beta \simeq 0.25$ was found. Hence, $\mathrm{U}_{\mathrm{s}} \ll+50 \mathrm{~V}$ for $\mathrm{U}_{\mathrm{gf}}=125 \mathrm{~V}$ and $\mathrm{U}_{\mathrm{f}}=100 \mathrm{~V}$. A slightly negative shield potential seems to be optimal with respect to high ion extraction efficiency and to prevent electrons from passing through the extraction aperture.

\section{E. Modulation}

It is, in principle, possible to modulate the MEG by variation of the shield electrode potential, in order to determine the residual current. However, the shield electrode potential is still influencing the electric field near the collector. The residual current will thus very likely be modulated together with the ions created from the residual gas. Hence, the results from modulation of the MEG are doubtful 15). It is, however, acceptable to subtract the $x-r a y ~ l i m i t$, once it is known, from pressure readings to obtain correct results even in the low $10^{-12}$ Torr range.

\section{CONCLUSIONS}

The design of a miniaturized extractor gauge MEG is presented and the influence of most of the geometrical and electronical parameters on its performance are discussed. The gauge fits in an aperture of $15.7 \mathrm{~mm}$ (less than $2 / 3$ inch). Its sensitivity is about $5 \mathrm{Torr}^{-1}$ for nitrogen at $10 \mathrm{~mA}$ emission current and slightly higher $\left(6\right.$ Torr $\left.^{-1}\right)$ at emission currents below $5 \mathrm{~mA}$. The MEG's low pressure limit, being mainly due to $x$-rays, was found at about $5 \times 10^{-12}$ Torr. Electronic ion desorption from gases, chemisorbed at the anode, and intrinsic outgassing scarcely affect its operation even at very low pressures: due to the small grid surface area, it is characterised by a high current density at the anode providing an even faster removal of chemisorbed gases than in gauges of normal size. The gauge may be operated at pressures up to $10^{-5}$ Torr. It is mounted at a metal flange with ceramic feedthroughs and may be operated by many standard Bayard-Alpert gauge control units. A prototype has already been operating for a period of over one month without any failures. 


\section{ACKNOWLEDGEMENT.}

The author is very much indebted to Mr. Jacques Place (CERN) whose skill in the construction of the various prototypes was an essential contribution to this work.

\section{REFERENCES}

1) E. Fischer, J. Vac. Sci. Technol. 9, 1203 (1972); and E. Fischer 6th Intl. Vac. Congr., Kyoto, 1974, to be published.

2) D. Blechschmidt, 6th Int1. Vac. Congr., Kyoto, 1974, to be published.

3) M.G. Mourad, T. Pauly and R.G. Herb, Rev. Sci. Instr. 35, 661 (1964).

4) D. Blechschmidt, J. Vac. Sci. Technol. 10, 376 (1973).

5) P.A. Redhead and J.P. Hobson, Brit. J. App1. Phys. 16, 1555 (1965).

6) P.A. Redhead, J. Vac. Sci. Techn. $\underline{3}, 173$ (1966).

7) A. Barz and P. Kocian, J. Vac. Sci. Technol. 7, 200 (1970).

8) U. Beek and G. Reich, J. Vac. Sci. Technol., 9, 126 (1972).

9) H.J. van Ark and J. van de Rotte, Vak. Techn. 17, 173 (1968).

10) J.J. Grodski and B.W. Schumacher, Rev. Sci. Instr. 39, 7C2 (1968).

11) B. Aingeril and Z. Hulek, J. Vac. Sci. Technol., 11, 461 (1974)

12) H. Samuel, Proc. Inst. Rad. Eng. 33, 233 (1945).

13) B.L. Schram, F.J. de Heer, M.J. van der Wiel and J. Kistemaker, Physica $\underline{31}, 94$ (1964).

14) L.G. Pittaway, J. Phys. D : App1. Phys. $\frac{3}{8}, 1113$ (1970), and

P.A. Redhead, J. Vac. Sci. Technol., 6 , 848 (1969).

15) P.A. Redhead, J. Vac. Sci. Technol. 4, 57 (1967). 
Dimensions of the MEG head (in $\mathrm{mm}$ ), the notations are explained in Fig. 3

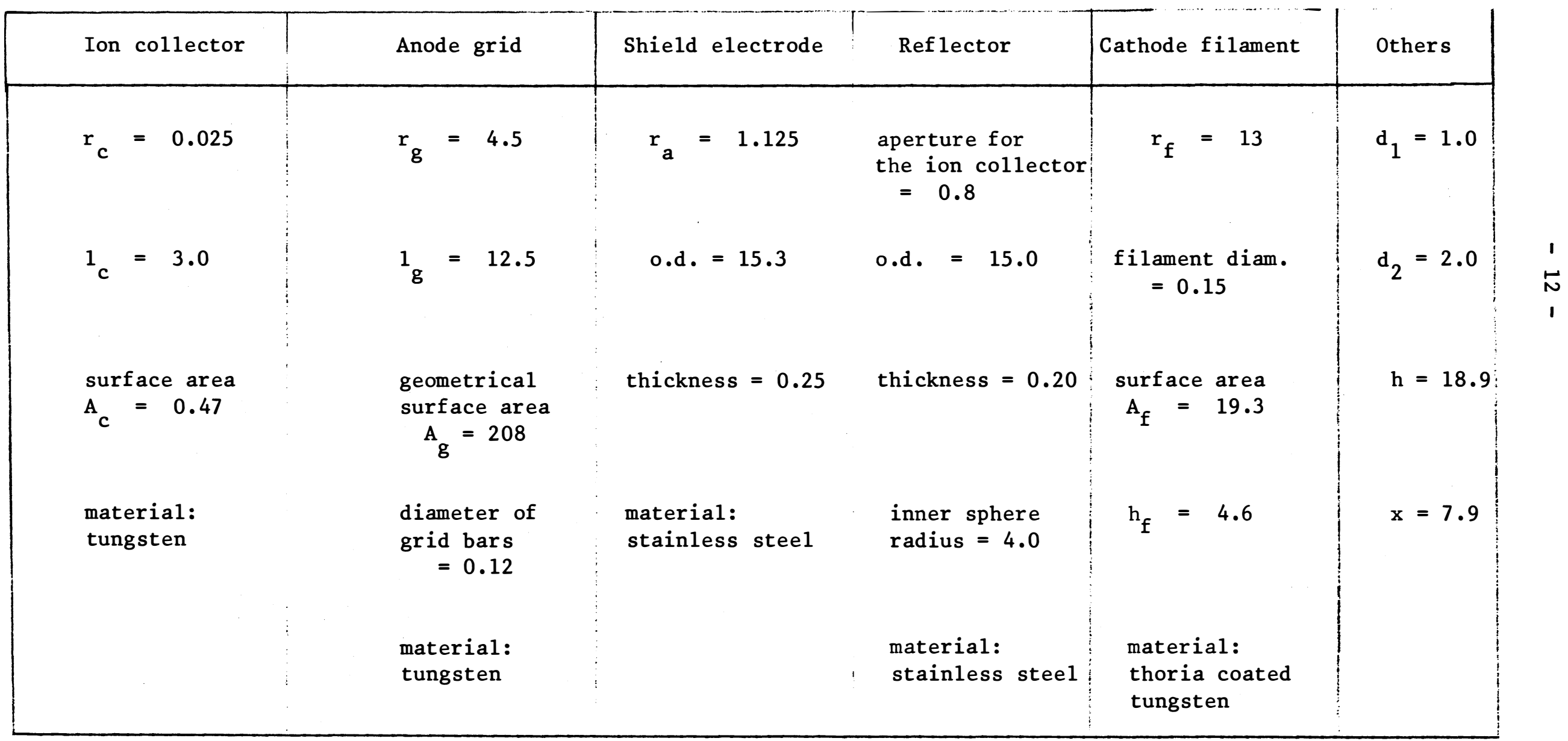


Table II

Operational data of the MEG

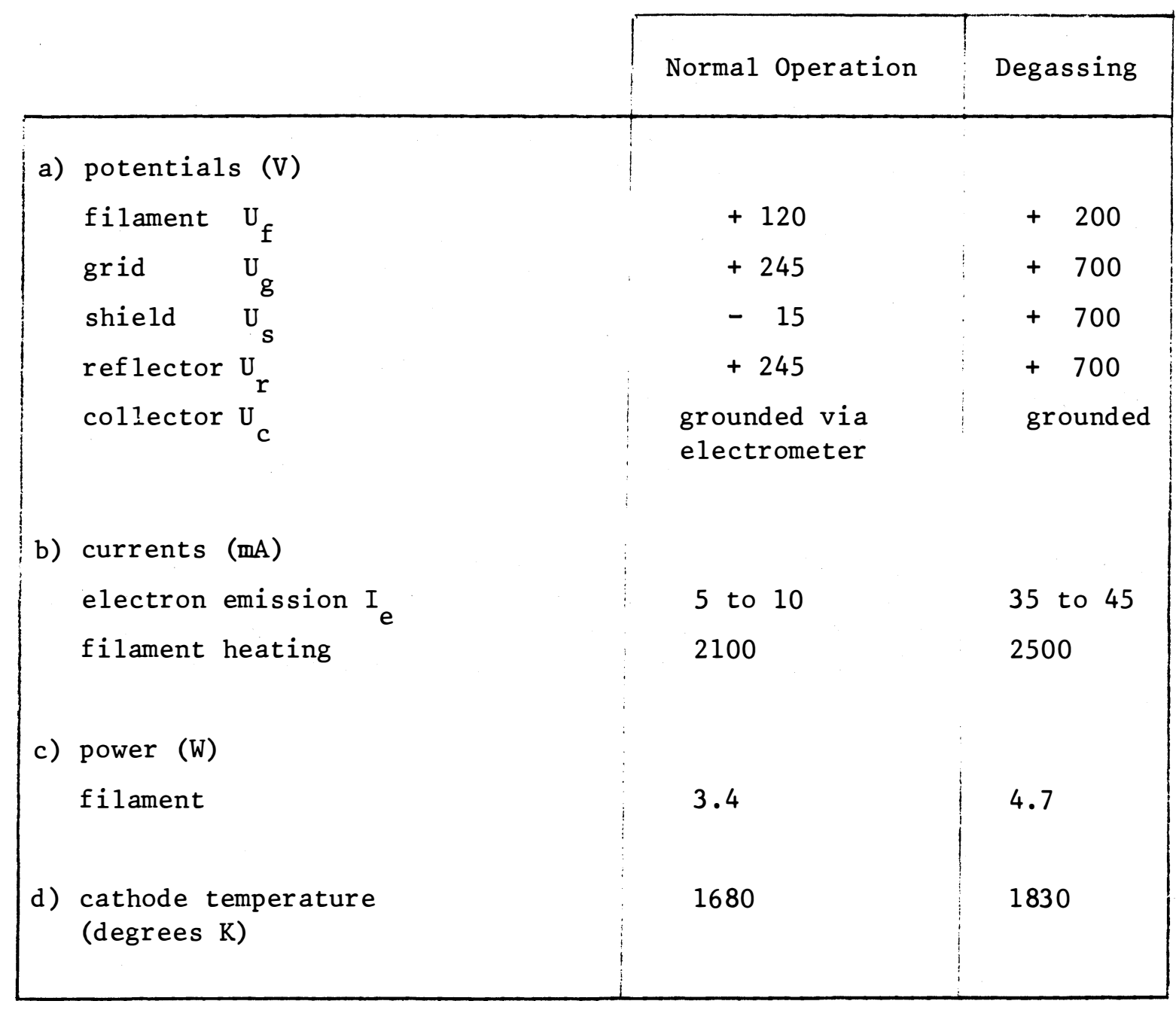




\section{Figure captions}

Fig. 1 Photograph of the MEG. The unit on the scale is $1 \mathrm{~cm}$.

Fig. 2 Cross-section and drawing of the MEG.

Fig. 3 Schematic drawing of the gauge head.

Fig. 4 The collector current $I_{c}$ as a function of the pressure of injected argon, nitrogen and hydrogen. The low pressure end of the curve (open dots) is obtained from a nitrogen injection (closed dots) subtracting the pressure independent residual current.

Fig. 5 The collector current $I$ of the MEG as a function of the electron emission $I^{C}$ for two different pressures. The $I$ - values for $p=1.5 \times 10^{-9}$ Torr are reduced by a factor of ten in this plot.

Fig. 6

The collector current $I$ as a function of the grid-tofilament potential $U_{g f}$ for different filament bias $U_{f}$ and shield potentials $U$ gf The measurements were performed at $1.7 \times 10^{-9}$ Torr $\mathrm{N}_{2}$-equiv.

Fig. 7 The collector current I plotted versus the gird-to-filament potential $U_{g f}$ for different cathode and shield potentials $\mathrm{U}_{f}$ and $\mathrm{U}_{\mathrm{s}}$ agf the parameters. The measurement was made at a nitrogên equivalent pressure of $5.5 \times 10^{-12}$ Torr. 

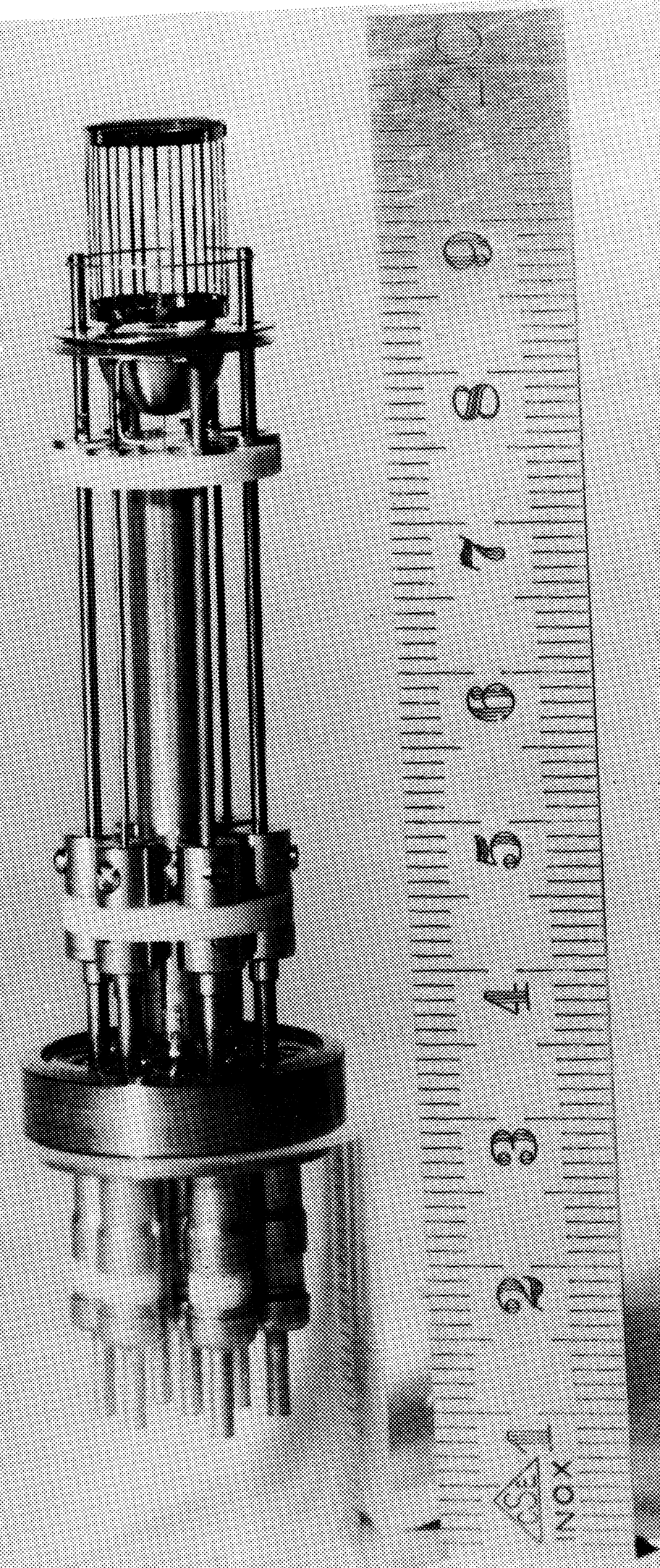

Fig. 1 


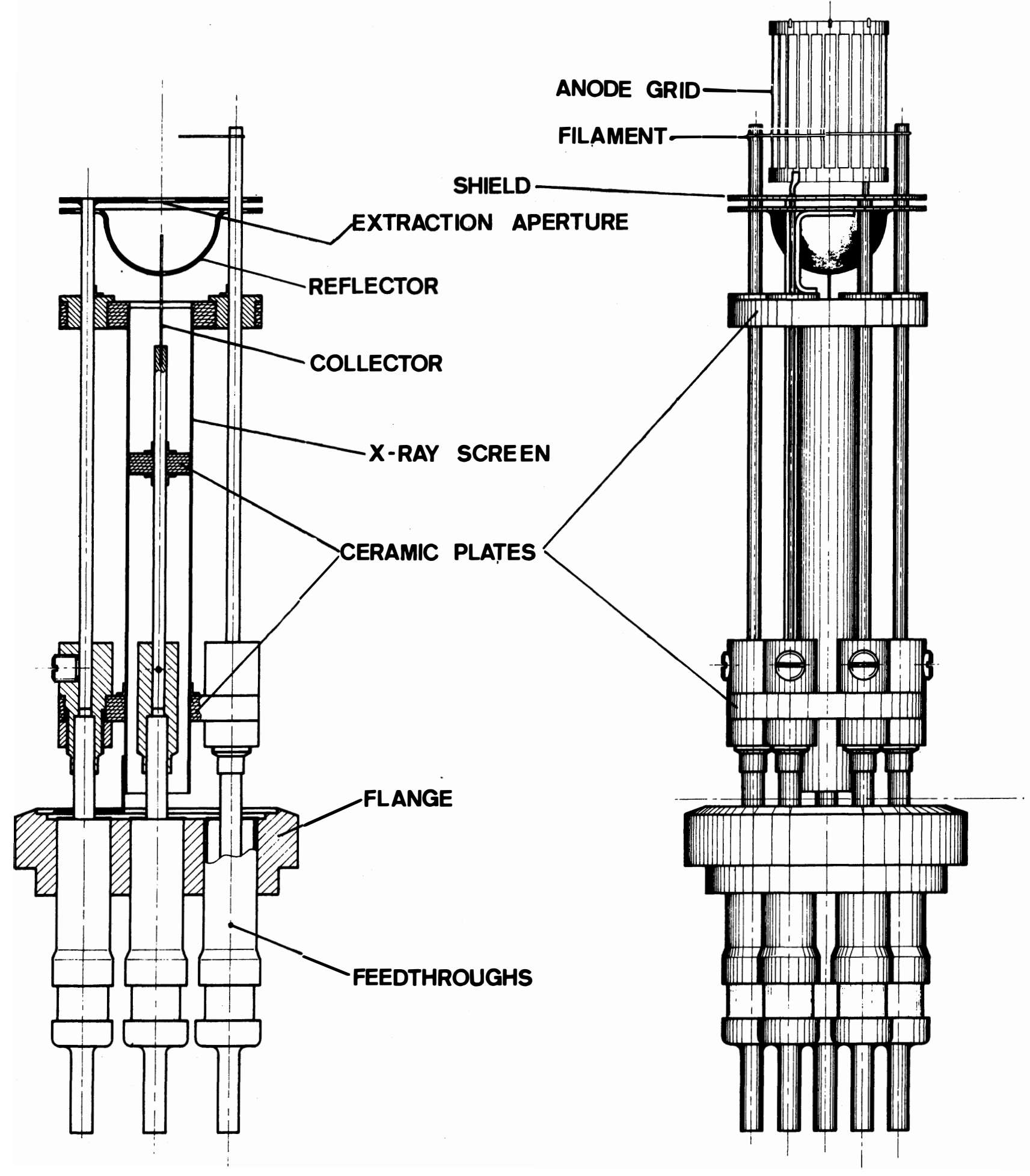

Fig. 2 


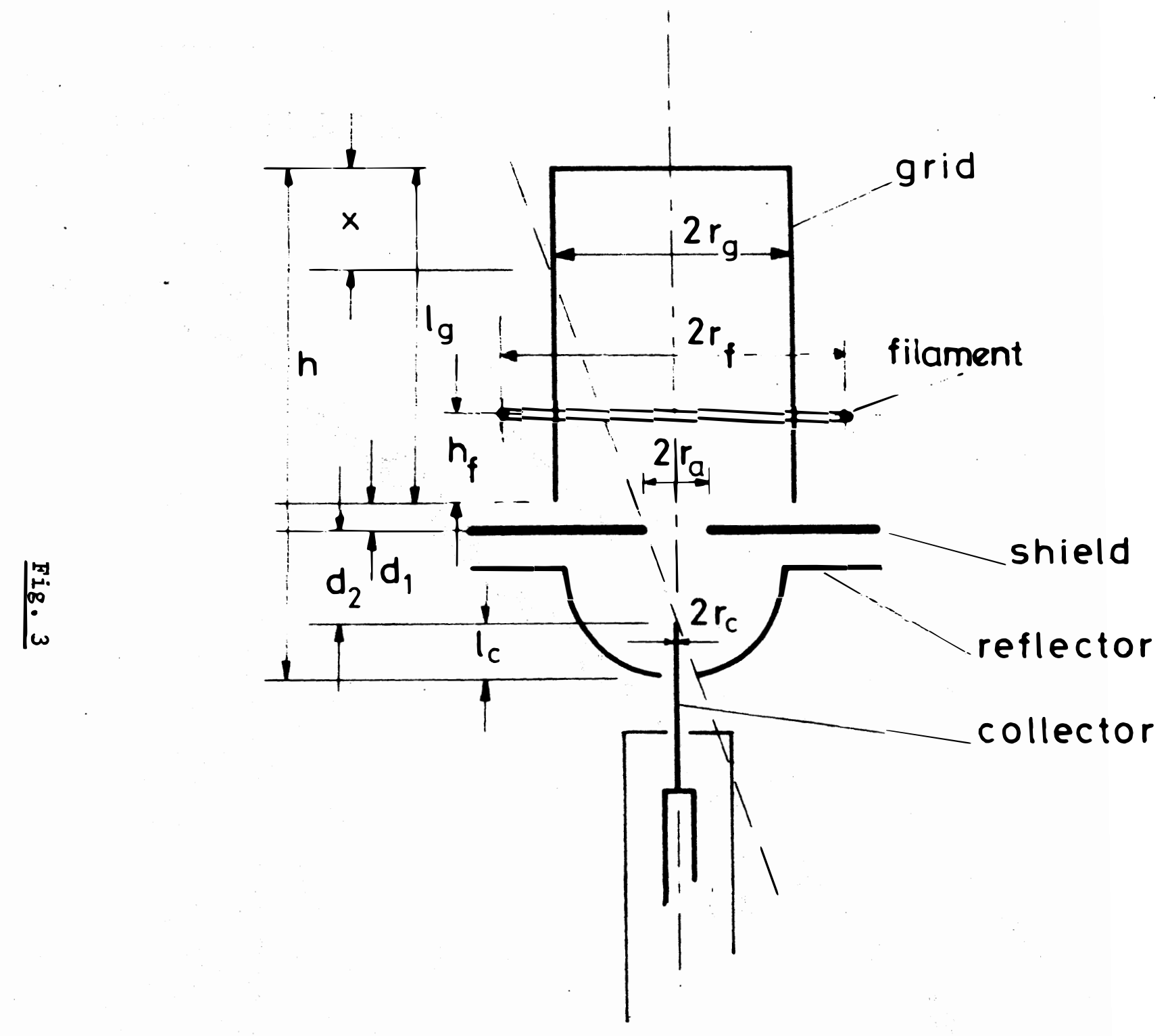




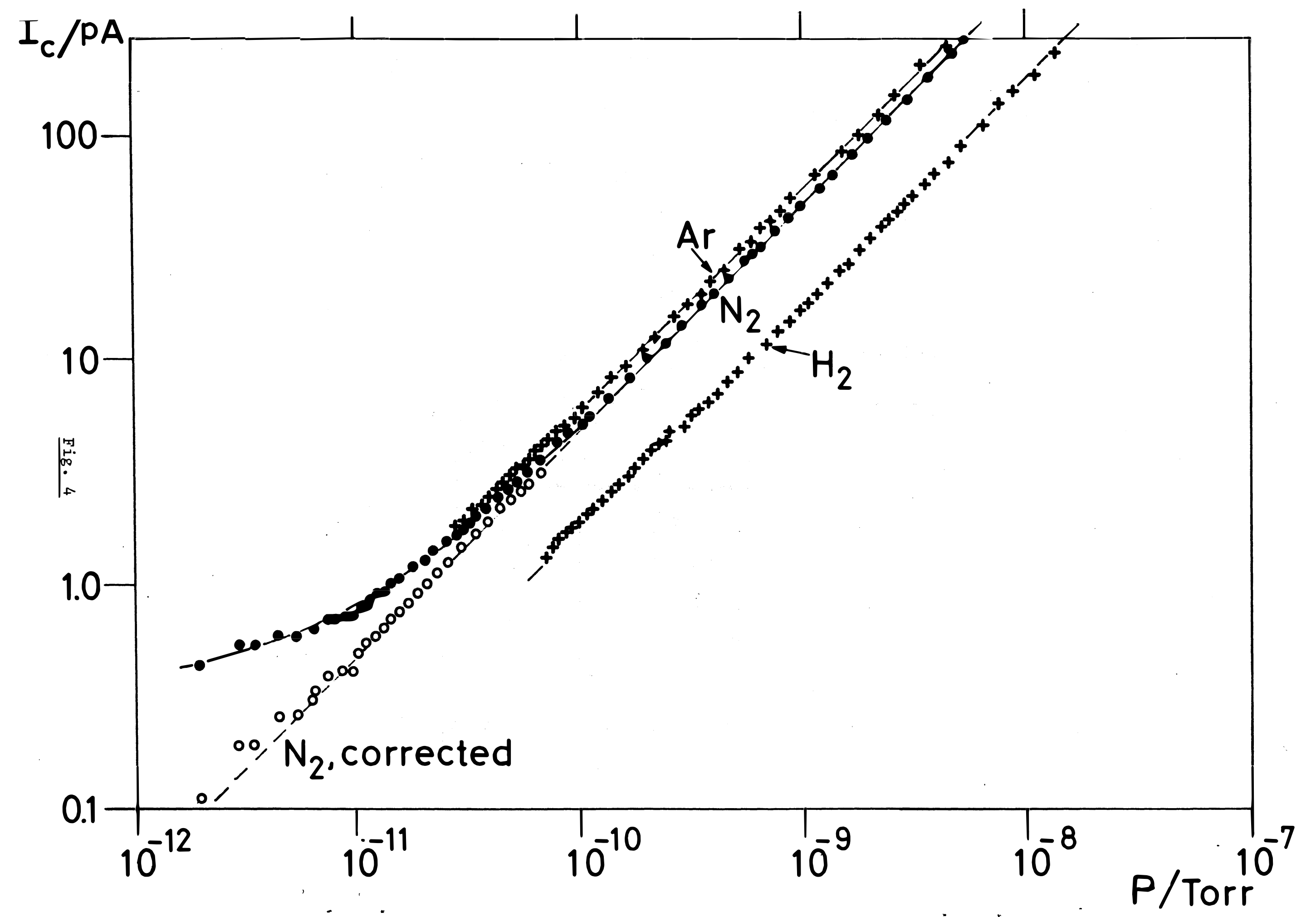




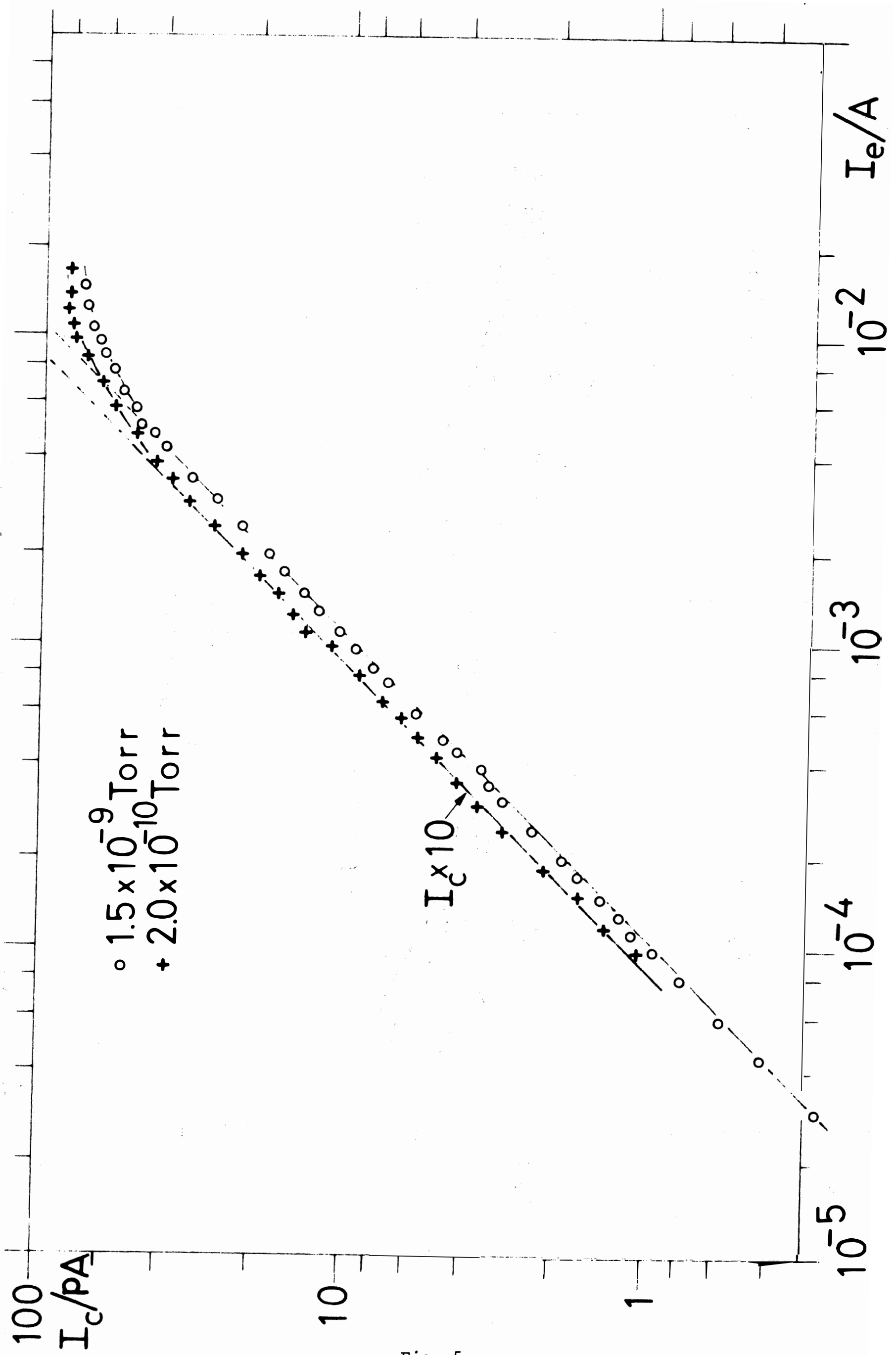

Fig. 5 


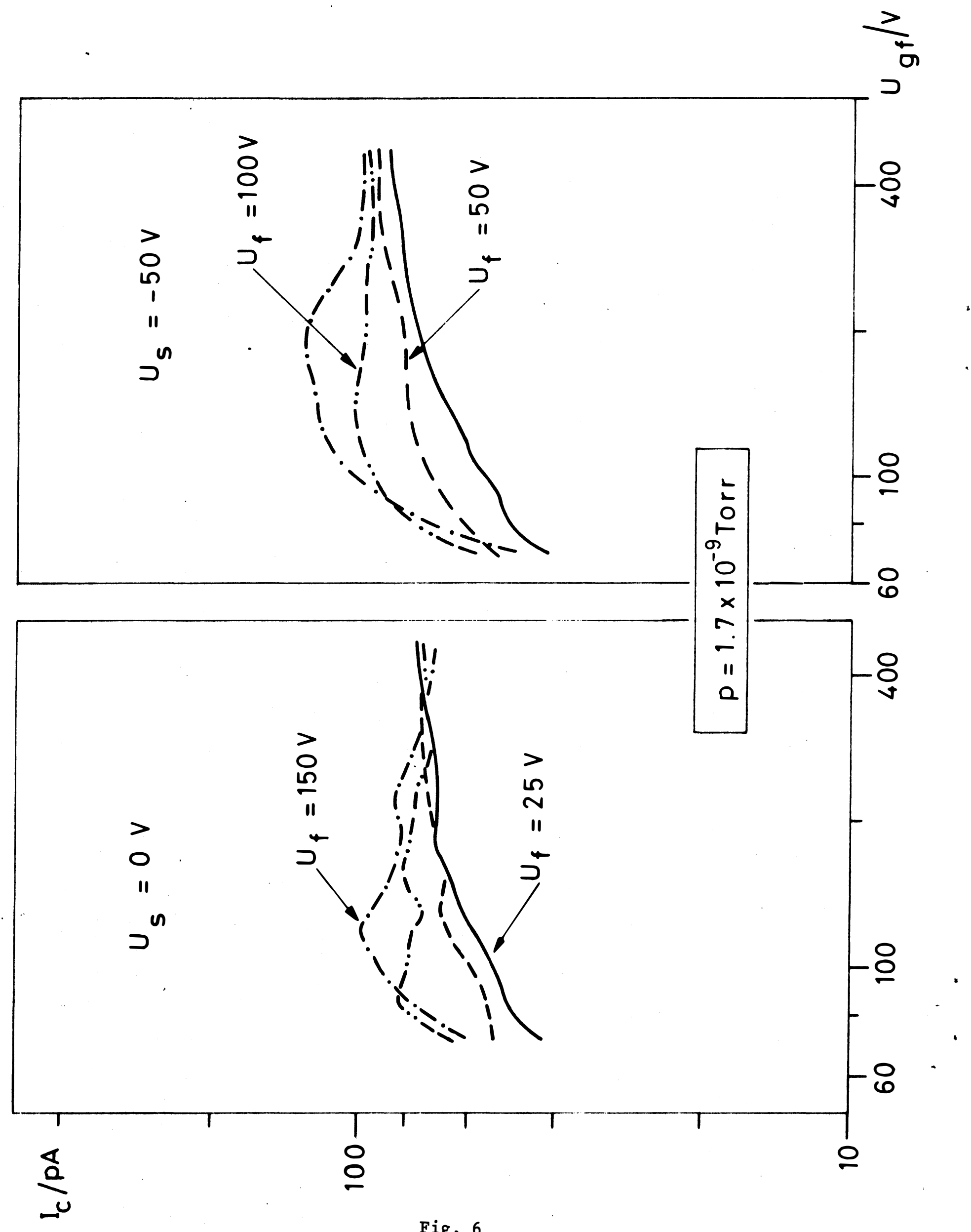

Fig. 6 


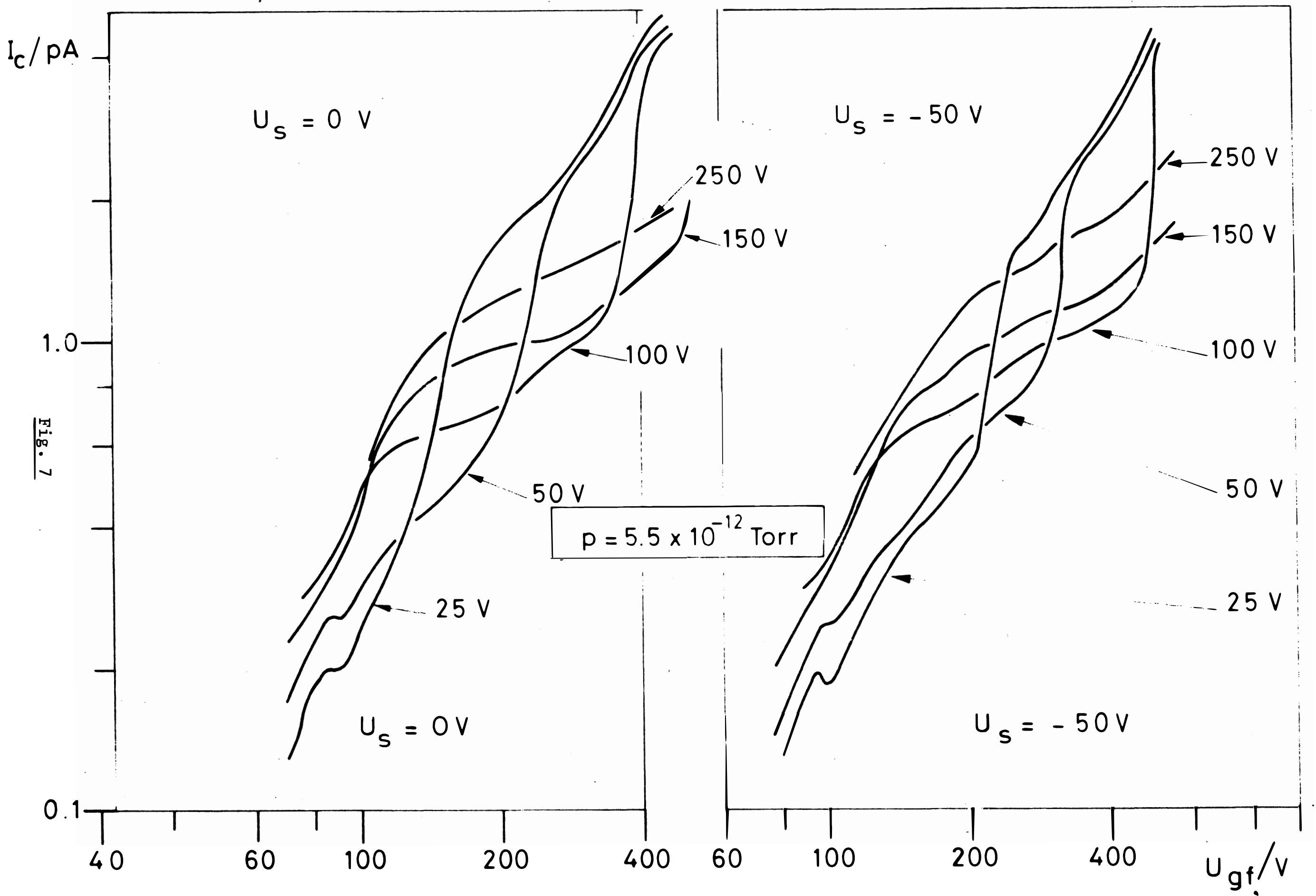

